\title{
„Wir begannen nicht im Jahre Null!" Bestseller, Autoren, Leser in Deutschland vor und nach 1945
}

\author{
"We did not start in the Year Zero" \\ Bestsellers, Writers, Readers in Germany before and after 1945
}

Christian Adam

\begin{abstract}
There was no 'zero hour' (Stunde Null) in German literature, though nazification and denazification had serious impact on the book market. The paper shows this by looking at bestselling books in East and West Germany before and after 1945. Entertaining books and factual novels were among the most successful books in the Third Reich. Freed of their political time reference, many of the texts from the Nazi era lived on after the end of the war. Both genres consequently left a long-term mark on the book market. Some authors like Karl Aloys Schenzinger were close to the National Socialist Party, others like Ehm Welk were critical to the regime.
\end{abstract}

\section{Keywords}

zero hour; nazification; denazification; mass literature; indexing; entertainment; propaganda; pre-censorship; Third Reich; East and West Germany 
Mit Blick auf das Jahr 1945 und die deutsche Literatur kann von einer ,Stunde Null“ nicht die Rede sein. Es gab - wie in allen anderen Lebensbereichen - keinen Neubeginn aus dem Nichts, keine tabula rasa. Diese Fiktion, dieser Traum hatte stets die Funktion, das, was davor war, ein totalitäres Regime, das mit einem verheerenden Krieg Europa und die Welt in eine Katastrophe gestürzt hatte, soweit es irgend ging, vergessen zu machen. Den Überlebenden sollte ein unbelasteter Neubeginn ermöglicht werden.

Dennoch war das Jahr 1945, vor allem aber auch die zwölf Jahre nationalsozialistischer Herrschaft, ein gewaltiger Einschnitt, eine tiefe Zäsur. Hier wurden Entwicklungen jäh abgebrochen, Lebenslinien zerschnitten, ging manches für immer verloren.

Mein besonderes Forschungsinteresse galt immer schon den populären Literatursegmenten, der Massenlektüre. Und so soll in diesem Text der Frage nach Zäsuren, aber auch Kontinuitätslinien, über 1945 hinweg vor allem anhand der viel gelesenen Literatur in Deutschland nachgegangen werden. Dabei werden notwendigerweise Autoren in den Blick kommen, deren Werke nicht zum unmittelbaren Kerngeschäft des Literaturwissenschaftlers zählen. Diese Erweiterung des Blicks verspricht aber gerade für mentalitätsgeschichtliche Fragestellungen einen großen Erkenntnisgewinn.

Im Sommer 1948 besuchte eine Kommission US-amerikanischer Verlagsvertreter das von den alliierten Siegermächten besetzte Deutschland. Sie sollten im Auftrag der amerikanischen Militärregierung einen Bericht über den Zustand der deutschen Buchbranche nach dem Zweiten Weltkrieg erstellen. Sie kamen zu dem Schluss, dass die Branche - die bis in die Dreißigerjahre hinein in vielen Bereichen als weltweit führend gegolten hatte - innerhalb eines kurzen Zeitraums zwei schwere Schläge erlitten habe: die ,Nazifizierung 1933 und die ,Entnazifizierung ${ }^{6} 1945 .{ }^{1}$ Dies machten sie vor allem an den in der Buchbranche tätigen Personen fest. Mit dem Machtantritt der Nationalsozialisten und der von ihnen betriebenen Gleichschaltung auch des Kulturbetriebs war ein ungeheurer - wir würden heute sagen - brain drain verbunden. Verleger, Autoren, Buchhändler, die politisch nicht auf der Linie der Nazis lagen, wurden aus dem Beruf gedrängt, bedroht, mundtot gemacht. Gleiches galt für alle Juden in der Branche, die zudem bald um ihr Leben fürchten mussten. Wer konnte, floh, viele wurden deportiert und ermordet. In die frei gewordenen Positionen (sowohl in den Verlagen aber auch unter den Autoren) rückten andere nach, oftmals aus der eher ,zweiten Reihe‘ kommend. So mussten beispielsweise Autoren von Weltruhm wie Heinrich und Thomas Mann oder Ricarda Huch aus der Akademie der Künste, Sektion Dichtkunst, austreten und dafür systemnahen Autoren wie Hanns Johst oder Hans Grimm Platz machen. In den Jahren danach versuchten die nationalsozialistischen Kulturlenker, allen voran der Propagandaminister Joseph Goebbels, einen systemkonformen Literaturmarkt und eine eigene NS-Literatur in Szene zu setzen. Mit mehr oder weniger Erfolg.

Ein ähnlicher Prozess sollte sich nun, nach der Kapitulation 1945, in Teilen wiederholen: Die Alliierten erlaubten nur solchen Verlegern und Verlagen die Wiederaufnahme der Tätigkeit, die als politisch unbescholten galten. Wieder ging - so das Fazit der

1 The Visiting Committee of American Book Publishers (hrsg.) (1948): German Book Publishing and Allied Subjects. Munich - New York: [ohne Verlag]. 
amerikanischen Sachverständigen - viel professionelles Know-how verloren. Gleiches galt für die Schriftsteller, die sich - wie viele andere erwachsene Deutsche auch - einer Entnazifizierung unterziehen mussten. Diese wurde in den Zonen unterschiedlich gehandhabt. Am radikalsten - so ist sich die Forschung einig - wohl in der sowjetischen und der amerikanischen Zone. Man hatte in Fragebögen Auskunft zu geben und ggf. vor sogenannten Spruchkammern zu erscheinen. Das Spektrum der dort gefällten Urteile konnte von ,hauptschuldig“ über ,belastet' und ,Mitläufer' bis zu ,entlastet' reichen.

Mit Blick auf diese ,Bruchkante‘ 1945 gilt es, in Deutschland den besonderen Fall zu betrachten, dass sich unmittelbar nach Kriegsende eine neue Trennlinie abzeichnete: die zwischen Ost und West, amerikanischem und sowjetischem Einflussbereich. Also steht nicht nur die Frage im Raum, welche Kontinuitäten und Brüche es gab, sondern wie wurde im Osten und wie im Westen mit dem jeweiligen Erbe umgegangen? Eine Vermutung zu Beginn der Untersuchungen war, dass im Osten, der späteren DDR, eher das ,strukturelle Erbe‘ der NS-Zeit angenommen wurde, in der Bundesrepublik (West) eher das personelle. Konkret gesagt: Die kommunistischen Machthaber werden möglicherweise bestimmte Methoden der Kontrolle übernommen haben, NS-belastete Autoren oder Verleger werden dagegen eher im Westen zu finden gewesen sein.

Zunächst soll diese Zäsur quantitativ in den Blick genommen werden. Für die Studien² zur Bestsellerliteratur in Deutschland vor und nach 1945 wurden im großen Umfang Absatzzahlen von Büchern ermittelt. Bei aller Vorsicht, die beim Umgang mit solchen Zahlen geboten ist, und bei aller Unvollständigkeit der Erhebung: Der Blick auf die viel verkaufte und dann vielleicht auch gelesene Literatur macht doch bestimmte Tendenzen erkennbar. Dies soll anhand einiger Schaubilder erläutert werden.

Zu diesem Zweck wurden die 100 meistverkauften Bücher in Gesamtdeutschland zwischen 1945 und 1961 aufgelistet und diese dann anhand ihrer Autoren kategorisiert (Schaubild 1). Über die Hälfte der bestverkauften Bücher ist entweder vor 1933 erstmals erschienen oder stammt von belasteten Autoren oder solchen der Inneren Emigration.

Lässt man die Übersetzungsliteratur außen vor, so ergibt sich ein noch eindeutigeres Bild: Dann sind nur noch 20 \% der hoch aufgelegten Titel in Nachkriegsdeutschland den literarischen Gegenstimmen oder der neuen deutschen Literatur zuzuordnen (Schaubild 2). Im Sektor der Massenlektüre können wir also durchaus von einem breiten Strom an Lesestoffen sprechen, der den Buchmarkt über die Jahre 1933 und 1945 hinweg dominierte. Und so kann es kaum verwundern, dass bei einer Erhebung der im Jahr 1947 in Leihbüchereien in Mecklenburg-Vorpommern (einem Landesteil der DDR) ähnliche Bücher als meistgelesene genannt wurden wie in den Westzonen: Edgar Wallace, Ludwig Ganghofer, Ehm Welk, Leo Tolstoi, Jack London, Karl May, Gottfried Keller, Maxim Gorki, John Knittel. ${ }^{3}$

2 Adam, Christian (2010): Lesen unter Hitler. Autoren, Bestseller, Leser im Dritten Reich. Berlin: Galiani. Sowie ders. (2016): Der Traum vom Jahre Null. Autoren, Bestseller, Leser: Die Neuordnung der Bücherwelt in Ost und West nach 1945. Berlin: Galiani.

3 vgl. Löffler, Dietrich (2011): Buch und Lesen in der DDR. Ein literatursoziologischer Rückblick. Berlin: Ch. Links, S. 327. 


\section{Die hundert meistverkauften Bücher in Nachkriegsdeutschland}

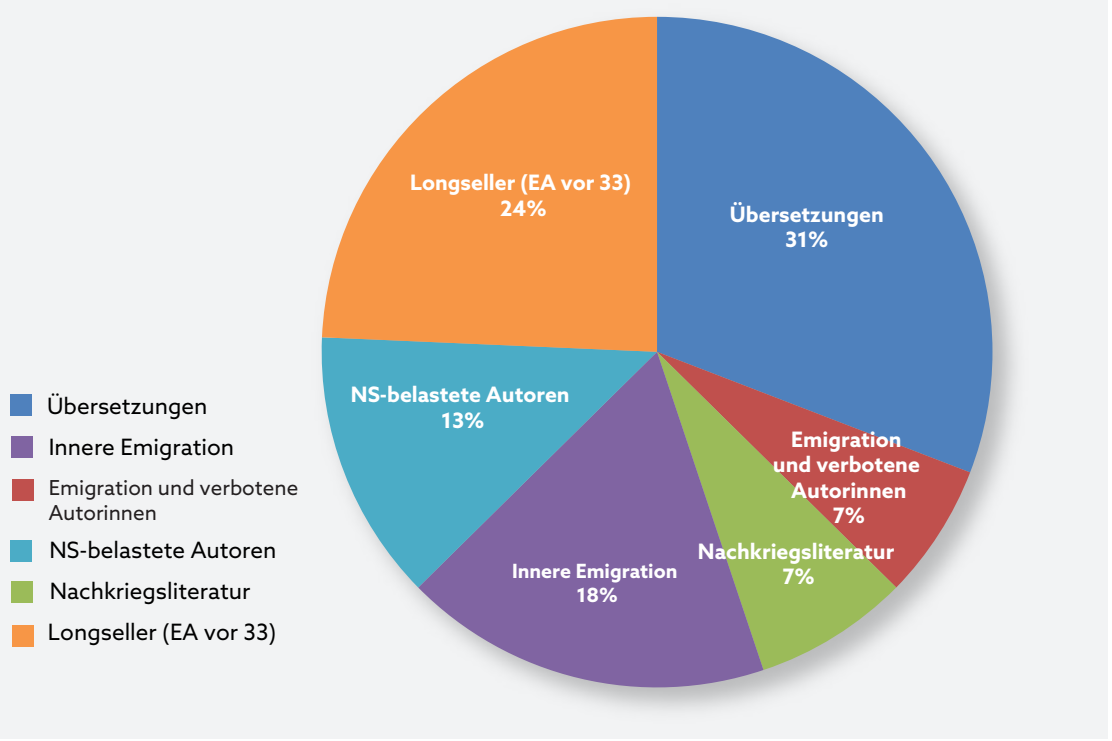

Schaubild 1: Die hundert meistverkauften Bücher in Nachkriegsdeutschland

\section{Literarische Tendenzen unter den meistverkauften Büchern (1945-1961)}

Gegenstimmen und neue Literatur

Longseller, Innere Emigration, Belastete

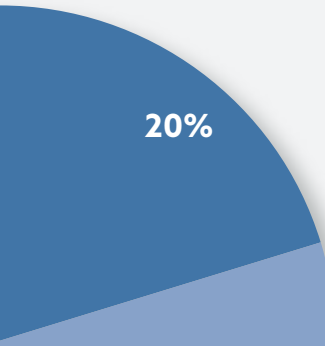

Schaubild 2: Die meistverkauften Bücher ohne Übersetzungsliteratur 
Diese Übersicht über die Top 100 der meistgelesenen Bücher ist hinsichtlich der Literatur in Ost- und Westdeutschland nicht unproblematisch. Je länger die deutsche Teilung währte, umso stärker bewegte sich die Lesekultur in den beiden deutschen Staaten in unterschiedliche Richtungen. Im Westen entwickelte sich ein freier Buchmarkt, im Osten sollte eine sozialistische Literatur gefördert werden. Und schon aufgrund der geringeren Einwohnerzahl und des kleineren Buchmarktes können nur wenige Bücher aus Ostdeutschland in dieser Liste der 100 meistverkauften Bücher enthalten sein.

Deshalb soll dieser quantitative Ansatz noch mit zwei weiteren Grafiken ergänzt werden (Schaubild 3). Diese Übersichten lassen deutliche Unterschiede zwischen DDR und Bundesrepublik hervortreten, markieren aber auch überraschende Übereinstimmungen. Ausgewertet wurden dabei die Literaturpreise, die in beiden deutschen Staaten zwischen 1945 und 1957 vergeben wurden. Deutlich wird, dass in Ostdeutschland sehr viel mehr Autoren aus dem Exil oder unter den Nazis verbotene Schriftsteller mit einem Preis bedacht wurden, während in Westdeutschland die Autoren der Inneren Emigration, die im Land gebliebenen Schriftsteller, eine größere Rolle spielten. Auch der Anteil der jungen, also der nach 1945 erstmals ins literarische Leben getretenen Autoren ist im Osten größer als im Westen. Bemerkenswert ist, dass der Anteil der systemnahen Autoren sich mit 9 bzw. 12 \% in einer ähnlichen Größenordnung bewegt. Wobei als Indikator für eine Systemnähe eine Mitgliedschaft in der Nationalsozialistischen Deutschen Arbeiterpartei (NSDAP) herangezogen wurde.

\section{Zwischen 1945 und 1957 vergebene Literaturpreise in Ostdeutschland}

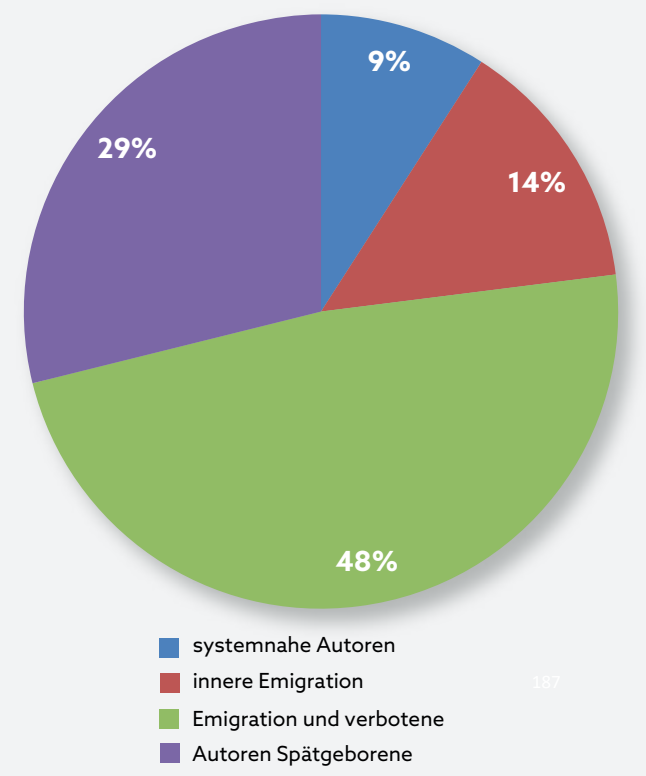

Zwischen 1945 und 1957 vergebene Literaturpreise in Westdeutschland

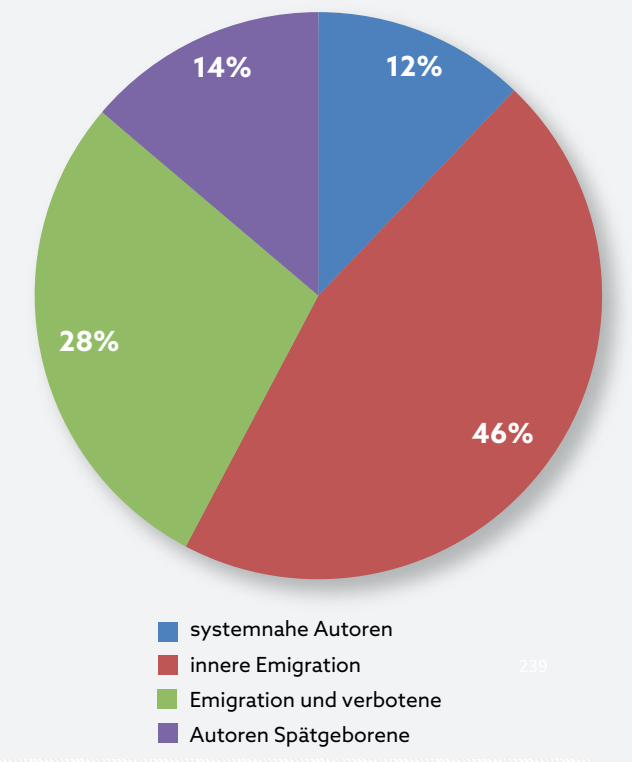

Schaubild 3: Literaturpreise in Ost- und Westdeutschland im Vergleich 
Allein die reinen Absatzzahlen sagen noch wenig darüber aus, was die Menschen wirklich lasen, noch weniger darüber, was sie dachten. Aber kann eine Beschäftigung mit solchen Zahlenspielen dennoch sinnvoll sein? Um diese Frage zu beantworten, muss man wissen, dass das Handeln sowohl der Nationalsozialisten bei der Machtübernahme als auch das der Alliierten nach der Kapitulation Hitler-Deutschlands von ähnlichen Prämissen ausging. Beide legten ihren Steuerungsversuchen für den Buchmarkt ein Kommunikationsmodell zugrunde, bei dem der, der bestimmt, was in Büchern steht, damit beeinflussen kann, was Menschen denken (Stimulus-Response-Modell). Folglich musste das Unerwünschte verboten und das Erwünschte gefördert werden. Beides geschah, was die konkreten Bücher anging, mit Verbots- und Empfehlungslisten.

So, wie die Nazis versucht hatten, das politisch oder aus anderen Gründen Missliebige zu verbieten, wollten die Alliierten die NS-Literatur zurückdrängen und damit die Voraussetzung für die Umerziehung der Deutschen schaffen. Auch der Stellenwert, den Amerikaner und Briten dem Buch als Medium zuschrieben, war dem vergleichbar, den ihm die Nazis eingeräumt hatten: Das Buch galt allen als das zentrale Medium, als die kulturelle Speerspitze. Man hoffte auch jetzt wieder, durch das Buch das Volk umerziehen zu können. Dabei war man von der Vermutung geleitet, dass man die für die Entnazifizierung besonders wichtigen Personenkreise - nämlich die besser Gebildeten und die ehemaligen Nazis - besonders gut erreichen könne: Beide Gruppen galten als besonders buchaffin. ${ }^{4}$

Was ist aber das Besondere am Medium Buch mit Blick auf solche Zäsuren und Steuerungsversuche? Zum einen das große Trägheitsmoment des Mediums. Wir kennen vor allem die Aktionen der Mächtigen, zum Beispiel die als Fanal inszenierten Bücherverbrennungen im Mai 1933 in Deutschland. Als symbolische Handlungen haben sie sich tief ins Gedächtnis eingegraben. Aber während man einen missliebigen Radiosender einfach abschalten kann oder einer Tageszeitung von heute auf morgen die Lizenz entzieht, lassen sich Bücher nicht so einfach verbieten und aus dem Verkehr ziehen. Auch das Verbotene kann im Privaten weiter existieren, weiter von Hand zu Hand gehen und gelesen werden. Insofern besitzt jedes Buch per se ein gewisses subversives Moment. Als im Krieg im Rahmen der Büchersammlung der NSDAP für die Deutsche Wehrmacht Buchspenden aus Privathaushalten für die Soldaten an der Front gesammelt werden sollten, mussten die Parteidienststellen feststellen, dass ein Großteil der gesammelten Bücher gar nicht verwendet werden konnte: Es handelte sich um verbotene Bücher, die noch in großer Zahl im Privatbesitz waren. Auch wirkte sich eine Indexierung auf Verkauf und Vertrieb nicht sofort aus. So erinnerte sich der Literaturkritiker Marcel Reich-Ranicki später, dass er in den Dreißigerjahren die Bücher des verfemten Autors Erich Kästner trotz Verbot für wenig Geld beim Antiquar in Berlin unterm Ladentisch kaufen konnte. ${ }^{5}$

4 vgl. Gruschka, Bernd R. (1995): Der gelenkte Buchmarkt. Die amerikanische Kommunikationspolitik in Bayern und der Aufstieg des Verlages Kurt Desch 1945 bis 1959. In: Archiv für die Geschichte des Buchwesens 44, S. 54 .

$5 \quad$ vgl. Reich-Ranicki, Marcel (2003): Mein Leben. München: dtv, S. 39. 
Dabei muss betont werden, dass die Zensur- und Verbotsmaßnahmen damit nicht verharmlost werden sollen; aber solche Maßnahmen waren immer auch durchlässig. Das galt im Übrigen auch für die Zeit nach dem Krieg, was die Lektüreeindrücke aus der Sowjetischen Besatzungszone 1947 belegen. Auch hier tauchten gern gelesene Bücher wie Edgar Wallace oder Karl May auf, die so nicht unbedingt ins Konzept der Mächtigen passten.

Mit Blick auf das Dritte Reich muss an dieser Stelle nochmals hervorgehoben werden, dass es dort - entgegen der weitverbreiteten Annahme - keine flächendeckende Vorzensur für Literatur gab. Eine Zensur vor Drucklegung existierte nur für bestimmte Literatursegmente wie das ausgesprochene Unterhaltungsschrifttum oder parteinahe Schriften. Die Lenkungsinstanzen setzten überwiegend auf die Schere im Kopf von Autoren und Verlegern. Auch wer fremdsprachige Literatur im Original lesen konnte, war lange Zeit im Vorteil: Während sich Aldous Huxleys dystopischer Roman Welt wohin? im Herbst 1935 auf der Liste [1] des schädlichen und unerwünschten Schrifttums ${ }^{6}$ wiederfand, war die englischsprachige Originalausgabe Brave New World bis 1939 in Deutschland frei verkäuflich. Auf diesem Weg war ein Teil der englischen und amerikanischen Literatur, dabei auch aktuelle Titel, im Deutschen Reich noch zugänglich. Erst mit dem Krieg gerieten die Literaturen der jeweiligen Kriegsgegner unter Verbot. Auch wurden im Rahmen der Papierbewirtschaftung die Zugriffsmöglichkeiten auf die Buchproduktion insgesamt allumfassender und kamen schließlich einer flächendeckenden Kontrolle nahe.

Im Folgenden sollen Zäsuren und Kontinuitäten rund um die Bruchkante 1945 noch mithilfe konkreter Beispiele anschaulich gemacht werden, darunter der Schriftsteller Ehm Welk. Seine Bücher Die Heiden von Kummerow ${ }^{7}$ und Die Gerechten von Kummerow ${ }^{8}$ waren schon im Dritten Reich Bestseller. Die Heiden waren 1937 erstmals erschienen und gehörten mit über 730.000 verkauften Exemplaren zu den erfolgreichsten Romanen in NS-Deutschland. Es handelte sich um gängige Unterhaltungsliteratur, die in einem fiktiven Dorf Kummerow irgendwo im Deutschen Reich der Kaiserzeit spielt. In Welks Lausbuben- und Schelmengeschichten werden die Sympathien des Lesers auf die Jugendlichen des Ortes rund um Martin Grambauer gelenkt. Aus der Welt der Erwachsenen ist Martins Vater Gottlieb eine zentrale Figur. Er gilt als welterfahren, denn er hat in Berlin gearbeitet, und ,fast schon intellektuell', denn er liest als einziger der Bauern des Ortes eine Zeitung. Gottlieb Grambauer begehrt gegen Kirche und Obrigkeit auf. Der antiklerikale Geist mag manchem nationalsozialistischen Kritiker gefallen haben. Allerdings sind alle Figuren Welks (auch die Kirchenleute) mit sehr viel Liebe und Sympathie gezeichnet.

Bei allen Anklängen an konventionelle Heimatliteratur - Welk denkt und schreibt anders und bleibt tatsächlich weit entfernt von Blut-und-Boden-Ideologie. Will man

6 Reichsschrifttumskammer (hrsg.) (1935): Liste [1] des schädlichen und unerwünschten Schrifttums. Berlin: Reichsdruckerei.

7 Welk, Ehm (1937): Die Heiden von Kummerow. Roman. Berlin: Ullstein [später: Deutscher Verlag].

8 Welk, Ehm (1943): Die Gerechten von Kummerow. Roman. Berlin: Deutscher Verlag. 
Widerständiges zwischen den Zeilen lesen - bei Ehm Welk ist es möglich. So doziert in einer Passage der Kantor Kannegießer vor versammelter Schulklasse:

„Ich sage euch, der Geist wird doch siegen, ganz gewiß immer dann, wenn sich ihm ein ehrliches Herz zugesellt. Und ganz bestimmt dann, wenn dazu auch noch Mut kommt. Nicht bloß der Mut, der dreinschlägt, sondern auch der Mut zum Bekennen. Das merkt euch, ihr Kummerower Faulpelze. [...] Der Mensch, der nach dem Großen strebt, kennt überhaupt nicht Feind und Freund. [...] Er kennt bloß schlechte und gute Menschen, aber die soll er nicht danach richten, wie sie zu ihm sind, sondern ob sie anderen Gutes oder Böses tun. ${ }^{* 9}$

Was für große und entlarvende Worte in so finsteren Zeiten.

Welk konnte seine Karriere nach 1945 als einer der wenigen gesamtdeutschen Autoren fortsetzen. Er lebte in der DDR, war dort Mitglied der Akademie der Künste und wurde 1954 mit dem Nationalpreis ausgezeichnet, aber auch in der Bundesrepublik wurden seine Bücher weiter verlegt und gelesen. 1967 wurden Die Heiden von Kummerow sogar in einer deutsch-deutschen Gemeinschaftsproduktion für das Kino verfilmt.

Dabei war Welk eher zufällig zum Bestsellerautor geworden: Seine Laufbahn als Journalist hatte 1934 ein jähes Ende genommen, weil er sich in einem Leitartikel kritisch zur Lage der gleichgeschalteten Presse und über Propagandaminister Joseph Goebbels geäußert hatte. Das Blatt, dessen Chefredakteur er war, wurde für drei Monate verboten, Ehm Welk kam mehrere Tage in Haft in das Konzentrationslager Oranienburg. ${ }^{10}$ Nach dem Berufsverbot blieb Welk nur die Flucht aufs Land. Dort begann er als Ghostwriter und Buchautor für seinen alten Verlag, Ullstein, tätig zu werden. Seine daraus entstandenen Romane wurden zum Erfolg. Ironie der Geschichte: Sein zweiter Roman, Die Gerechten von Kummerow, erhielt 1944 sogar noch den 1. Preis des Reichsministeriums für Volksaufklärung und Propaganda für Unterhaltungsschrifttum ${ }^{11}$, der Dissident und Abweichler also als von Goebbels Ausgezeichneter - allerdings durfte über die Preisverleihung in der Presse nicht mehr berichtet werden, was aber eher nichts mit Welks politischer Vergangenheit zu tun hatte.

Welks Texte passten zu der von Joseph Goebbels propagierten Funktion von Unterhaltungsliteratur: Sie sollte - so der Propagandaminister - den Menschen Ablenkung vom Kriegsalltag bringen und allzu viele Anspielungen auf Politisches vermeiden. Ohne diese Bezugnahmen auf das Gegenwartsgeschehen oder gar die Partei unterlagen Welks Texte zudem keiner Vorzensur, das kam dem zunächst regimekritischen Autor Welk und seinem Verlag sicher ebenfalls entgegen. Der nach wie vor privatwirtschaftlich organisierte Buchmarkt und letztlich die Leserinnen und Leser nahmen die Bücher dankbar auf und führten sie zum Erfolg. Mit ihren leicht kritischen Untertönen gegenüber der Obrigkeit erfüllten die Texte zugleich eine Art Ventilfunktion. Beides - die Zeitlosigkeit und das Entlastende - machten Die Heiden von Kummerow auch über 1945 hinaus an-

9 Welk [Anm. 7], S. 188.

10 vgl. Reich, Konrad (2008): Ehm Welk. Der Heide von Kummerow. Die Zeit. Das Leben. Rostock: Hinstorff, S. 191-193.

11 Ullstein Archiv [Berlin]: Deutscher Verlag, Bericht über wichtige Geschäftsvorfälle im Oktober 1944. 
schlussfähig; in beiden deutschen Staaten und unterschiedlichen Systemen. So untadelig man Ehm Welk als Person beurteilen mag: Seine Literatur wirkte in der NS-Zeit durchaus systemstabilisierend, gerade weil sie nicht systemnah war, also auch Leser erreichte, die vordergründige Propagandaliteratur abgelehnt hätten.

An Welk lässt sich mit Blick auf die Zäsur 1945 etwas zugespitzt sagen, dass trotz aller Verbots- und Umerziehungsmaßnahmen der alliierten Siegermächte die NS-Literaturpolitik ihre Wirkung weit über das Regimeende hinaus entfalten konnte. Der große Erfolg vermeintlich zeitloser, unpolitischer Mainstream-Unterhaltung in den Fünfziger- und Sechzigerjahren entsprang eben nicht nur dem Lesebedürfnis der Zeitgenossen, sondern war so gesehen eine Spätfolge Goebbelsscher Politik, der genau solche Literatur begünstigt hatte. Text und Autor an sich waren den alliierten Kontrollinstanzen dabei zu Recht völlig unverdächtig. Sie waren trotz aller harten Eingriffe und Brüche Teil jenes breiten Stroms an Literatur, der eingangs anhand von statistischem Material skizziert wurde.

Der andere Autor, der kurz vorgestellt werden soll, hat nicht wegen seiner Unauffälligkeit seinen Weg im Literaturmarkt nach 1945 fortsetzen können, sondern trotz seiner Regimenähe. Und gerade das macht ihn umso exemplarischer. Sein Tatsachenroman Anilin $^{12}$ gehörte zu den bestverkauften erzählenden Texten im Dritten Reich: Knapp eine Million Exemplare gingen bis 1945 über den Ladentisch. Und die Rezeption des Buches erlitt in Westdeutschland keinen Bruch: 1951 waren über 1,6 Millionen Bücher verkauft. Anilin gehörte in die Reihe der Rohstoff-Romane, die Sachbuchthemen verbunden mit einer romanhaften Handlung unter die Leute brachten.

Am Anfang der Erzählung steht der deutsche Chemiker Friedlieb Ferdinand Runge und seine Entdeckung des künstlich herstellbaren Anilin-Farbstoffes. Die Tragik seines Lebens (und auch die der Figur in Schenzingers Buch) besteht darin, zu Lebzeiten kaum Anerkennung für seine Leistungen erfahren zu haben. Kontrastiert wird die Geschichte seiner Entdeckung mit Erzählungen über den aus einer Pflanze gewonnenen Farbstoff Indigo. In Indien werden diese Pflanzen unter für die Arbeiter unmenschlichen Bedingungen angebaut und nach Europa verschifft. Dort verdienen nur die Importeure, vor allem durch Spekulation an der Rohstoffbörse in London. Der nationalistische Subtext in Schenzingers Roman kontrastiert englisches Spekulantentum mit deutschem Erfindergeist: Hier der Drang zur Gewinnmaximierung an den Börsen, dort der Wunsch, den technischen Fortschritt zum Wohl der Menschheit zu nutzen. Im Buch skizziert Schenzinger mit kühnem Strich die Geschichte der Entdeckung der Teerfarbstoffe und in ihrer Folge die Geburt der modernen chemischen Industrie, in der deutsche Werke und deutsche Forscher eine Schlüsselstellung einnehmen. Das Buch gipfelt in der Gründung der I.G. Farben, der Interessengemeinschaft Farbenindustrie AG, dem zeitweise größten Chemie- und Pharmakonzern der Welt. Der Leser folgt den Protagonisten schließlich auf dem Weg in ein neues Deutschland, in dem im Roman zwar nicht die Hakenkreuzfahnen flattern und auch kein ,Führer' grüßt, aber das klar als das Reich der Nationalsozialisten zu erkennen ist. Das Buch ist nicht frei von antisemitischen

12 Schenzinger, Karl Aloys (1937): Anilin. Roman. Berlin: Zeitgeschichte-Verlag Wilhelm Andermann. 
Anspielungen, wenn etwa eine Handelsorganisation beschrieben wird, die die Verbreitung eines Malaria-Medikaments verhindern will und der „vorwiegend Händler angehörten, die aus ihrem Blut und ihrer Gesinnung heraus dem neuen Deutschland nicht gewogen waren"13. Alle Erfindungen, die sich am Ende als segensreich für die Weltbevölkerung erweisen, wurden von deutschen Forschern gemacht. Künstliche Farbstoffe werden entgegen den Interessen der englischen Kolonialmacht entwickelt und machen Farben für jedermann erschwinglich. Obwohl die Siegermächte des Ersten Weltkrieges massenhaft deutsche Patente verletzen, forschen die kühnen Wissenschaftler weiter. Der Text transportierte also eine klare, propagandistische Botschaft, diente der technischen Mobilmachung und entsprach den Vorstellungen eines Teils der Nationalsozialisten, dass mit sogenannter ,weicher Propaganda' mehr zu erreichen sei, als mit explizit programmatischen politischen Schriften.

Der Text konnte nach 1945 mit nur wenigen Eingriffen für das westdeutsche Nachkriegspublikum tauglich gemacht werden. So wurde das dem Roman vorangestellte Zitat des „Reichsministers Dr. Frick“ gestrichen, in dem in der Erstausgabe vom „rassisch gesunden und unverbrauchten Volk" 14 die Rede war. Alle Hinweise auf die I.G. Farben, die durch verschiedene Unternehmensteile in den Völkermord verstrickt war, waren nun getilgt. Ebenfalls sucht man abfällige Bemerkungen über die Siegermächte des Ersten Weltkrieges, insbesondere die Engländer (die jetzt auch wieder auf der Siegerseite saßen), in der Nachkriegsausgabe vergeblich. Ein Satz wie: „Wir sind eingeengt, geographisch, wirtschaftlich, politisch. Wir wollen leben!" 15 hatte 1949 ausgedient. Andere Akzente waren leicht, aber entscheidend verschoben. So hieß es vor 1945: „Der künstliche Werkstoff ist zur deutschen Lebensfrage geworden." ${ }^{16}$ Nunmehr unter alliierter Kontrolle ganz im Sinne des Wiederaufbaus: „Der künstliche Werkstoff bedingt heute die Zukunft der deutschen Wirtschaft. ${ }^{\text {17 }}$

Nicht nur der Text, sondern auch der Autor musste sich einem Entnazifizierungsverfahren unterziehen. Hatte er doch, neben Anilin, auch den Roman Hitlerjunge Quex ${ }^{18}$ geschrieben, ein lupenreiner Propagandastoff, der erfolgreich verfilmt worden war. Der Uraufführung des Streifens im September 1933 hatte Hitler persönlich beigewohnt. Schenzinger war nie Mitglied der NSDAP gewesen, allerdings hatte ihm die Hitlerjugend für seinen Roman Quex das goldene HJ-Abzeichen verliehen. ${ }^{19}$ Vor der Spruchkammer kam Schenzinger 1948 mit einem blauen Auge davon: Er wurde lediglich als Mitläufer eingestuft. Er hatte eine Geldsühne und die Verfahrenskosten, insgesamt rund 230 Mark, zu tragen.

13 Schenzinger [Anm. 12], S. 297.

14 Schenzinger [Anm. 12], S. 5.

15 Schenzinger [Anm. 12], S. 375.

16 Schenzinger [Anm. 12], S. 375.

17 Schenzinger, Karl Aloys (1949): Anilin. Roman. München: Wilhelm Andermann, S. 378.

18 Schenzinger, Karl Aloys (1932): Der Hitlerjunge Quex. Berlin: Zeitgeschichte-Verlag Wilhelm Andermann.

19 Siehe Staatsarchiv Landshut: Spruchkammerakten zu Karl Aloys Schenzinger, Spruchkammer Landau Nr. 2664. 
Auch Anilin ist ein gutes Beispiel dafür, wie die NS-Literaturpolitik selbst den Grundstein dafür legte, dass viele literarische Stoffe ihre Karrieren nach 1945 ungebrochen fortsetzen konnten. Vor allem Goebbels und seine Leute hatten im Krieg apolitische Stoffe gefördert. Das machte die Texte in der Zeit nach der Nazi-Herrschaft anschlussfähig. Schenzinger konnte das vordergründig Unpolitische, das einige seiner Bücher im Nationalsozialismus so erfolgreich gemacht hatte, ohne Probleme während des Spruchkammerverfahrens für seine Verteidigung nutzen. So führte sein Anwalt im Zuge des Verfahrens aus, Schenzinger habe es abgelehnt

„parteigefällige Romane zu schreiben, dafür aber die bekannten Bücher Anilin und Metall [...], die alles eher als parteigefällig waren. Es wurde ihm deswegen von Verlegern und Buchhändlern ein völliger Misserfolg dieser Bücher vorausgesagt. Dass sie trotzdem Millionen Auflagen erlebten, lag auf anderem Gebiet. “20

Ehm Welk und Karl Aloys Schenzinger sind mit ihren Nachkriegskarrieren in gewisser Weise typisch für den bald in Ost und West geteilten deutschen Buchmarkt. So stehen beide eher für die Fortsetzung des Mainstreams, nicht für Innovationen oder einen Neuanfang. Sie sind also beispielhaft für Kontinuität, Schenzinger im Westen, Ehm Welk in West und Ost. Dabei warb der ostdeutsche Staat stärker um Emigranten und bot letztlich - das hatte auch der Blick auf die Literaturpreise in beiden deutschen Staaten gezeigt - den jungen Autoren zunächst statistisch gesehen mehr Raum.

Insofern landete Ehm Welk mit seiner widerständigen Vergangenheit folgerichtig in der sowjetischen Besatzungszone, wo der Autor 1946 sogar Gründungsdirektor der Schweriner Volkshochschule war. ${ }^{21}$ Dagegen musste ein vor 1945 so stark auf Seiten des NS-Regimes engagierter Autor wie Schenzinger seine Zukunft eher im Westen suchen. Insofern legt dieses Beispiel nahe, dass die westdeutsche Bundesrepublik eher das personelle Erbe (in Form der bekannteren und exponierteren Autoren und Autorinnen) antrat. Wobei der ähnlich hohe Anteil an ehemaligen Parteimitgliedern unter den Preisträgern in Ost und West zunächst erstaunen mag.

Dabei hatte man auch in der sowjetischen Besatzungszone, der späteren DDR, durchaus ein Bewusstsein dafür, dass man nicht völlig ohne die alten Eliten auskommen konnte. So wurde in der DDR eigens ein Verlag gegründet, der sich programmatisch auf die Fahnen geschrieben hatte,

„[...] mit allen seinen Publikationen dazu bei[zu]tragen, die Schichten des Mittelstandes und der ehemaligen Angehörigen der NSDAP sowie die früheren Offiziere und Berufssoldaten

20 Brief von Rechtsanwalt Fritz Höchtl an Spruchkammer Landau vom 31. März 1948. In: Spruchkammerakten zu Karl Aloys Schenzinger, Staatsarchiv Landshut, Spruchkammer Landau Nr. 2664.

21 Fritzlar, Sigrid (2011): Ehm Welk und der beschwerliche Aufbau der Volkshochule Schwerin vor 65 Jahren. Archivalie des Monats Mai 2011. In: Landesarchiv Mecklenburg-Vorpommern (hrsg.): Archivalie des Monats. https://www.kulturwerte-mv.de/Landesarchiv/Archivalien/Bisherige-Beitr\%C3\%A4ge/2011-05-EhmWelk-und-die-Volkshochschule-Schwerin/ (22. 9. 2019). 
der einstigen Hitlerarmee für die Festigung und Sicherung des Bündnisses aller werktätigen Schichten [mit der sozialistischen Arbeiterpartei ...] zu gewinnen. “22

Es war der Verlag der Nation, der Parteiverlag der National-Demokratischen Partei Deutschlands (NDPD). Zur Aufgabe des Verlags gehörte die

„Darstellung typischer Lebenswege und Entwicklungsprozesse von Angehörigen einzelner Schichten des Mittelstandes sowie der politischen Wandlung ehemaliger Mitglieder der NSDAP, Offiziere und Berufssoldaten der Hitlerarmee, auch in biografischer und autobiografischer Form. “23

Es war also ein Verlag, der mit seinen Büchern ganz bewusst die Wandlung ehemaliger Parteigänger der NSDAP zu guten sozialistischen Bürgern begleiten und befördern sollte.

Während sich aber im Westen bald ein von staatlichen Eingriffen freier Buchmarkt entfaltet hatte, etablierten die Machthaber in der DDR ein immer weiter ausgefeiltes Steuerungssystem. Hatte es im Nationalsozialismus zunächst kein flächendeckendes Zensursystem gegeben, so wurde dies im Krieg im Zuge der sogenannten Papierbewirtschaftung eingeführt. In der DDR konnte ebenfalls kein Buch ohne Druckgenehmigung erscheinen: Die Vorzensur war damit allumfassend. Der sozialistische Staat hatte so gesehen das Lenkungssystem der Nationalsozialisten fortgeführt und zur Perfektion gebracht.

Wo sich beide deutsche Staaten auf erschreckende Weise ähnlich waren, war darin, Eingriffe in die freie Meinungsäußerung hinzunehmen oder sogar von staatlichen Stellen durchführen zu lassen - bis hin zur Vernichtung und dem Verbrennen von Büchern. Dies geschah häufig im Kampf gegen sogenannte Schmutz- und Schundliteratur oder in der Auseinandersetzung mit dem ideologischen Gegner - auf beiden Seiten des Eisernen Vorhanges. Die SED ließ junge Pioniere Bücher verbrennen, ohne dabei etwas Anstößiges zu finden, im Westen wurde ebenfalls massenhaft Gedrucktes makuliert beides im Kampf gegen den neu erklärten politischen Gegner. Insofern zeigte sich auch hier ein erstaunliches Beharrungsvermögen des Systems und vor allem der am System beteiligten Personen.

Doch zurück zum Besuch der amerikanischen Verleger in Nachkriegsdeutschland. Sie mussten in ihrem Bericht an die Militärregierung eingestehen, dass es zunächst wenig innovative, neue Ansätze in der Buchbranche zu entdecken gab. Etwas resigniert hielten sie fest, dass so „wie die Buchindustrie einst gezwungen gewesen sei, Hitler zu dienen, diene sie sich jetzt dem neuen Zeitgeist und den amerikanischen Umerziehungszielen“24

22 Schreiben von Günter Hofé an die Abteilung Literatur und Buchwesen beim Ministerium für Kultur, Dr. Häckel, vom 13. 1. 1960. Übersendung der Leitsätze für den Verlag der Nation, S. 3. In: Bundesarchiv, Standort Berlin: BArch, DR 1/16217.

23 Bundesarchiv, Standort Berlin [Anm. 22], S. 4.

24 The Visiting Committee of American Book Publishers [Anm. 1], S. 23. 
an. Aber eben nicht aus Überzeugung, sondern leider nur aus Pragmatismus. Die Umerziehung schien zunächst also vor allem äußerlich zu wirken.

Was kann nun aber die Lehre aus der Betrachtung des Jahres 1945 mit dem Fokus auf der deutschen Literatur sein? Welche Herangehensweisen an Bruchkanten und Kontinuitäten erweisen sich als fruchtbar?

Dazu sei abschließend der deutsche Autor Friedrich Christian Delius zitiert. Dieser hatte in einem Vortrag über die Tücken des autobiografischen Erzählens ausgeführt:

„Lüge fängt da an, wo wir alles auf 0 oder 1, auf Ja oder Nein, auf Gut oder Böse herunterbrechen. Dagegen: Mit Sprache, ihrer Mischung aus Genauigkeit, Vieldeutigkeit und Poesie, mit Differenzierungskunst und Empathie lässt sich die Welt viel besser erfassen. ${ }^{\text {25 }}$

Kurz gesagt, die Vereinfachung ist der Feind der Wahrheit. Auch die geträumte ,Stunde Null', die tabula rasa war der Versuch zu einer solchen Vereinfachung. In der Realität war alles viel komplizierter. Es gab kein Schwarz und Weiß, sondern alle erdenklichen Zwischentöne. In der Realität konnte ein Buch von einem regimekritischen Autor durchaus systemstabilisierend sein und dennoch Autor und Werk zu Recht ihr Leben im Buchmarkt nach 1945 fortsetzen. In der Realität konnte sich parallel dazu ein anderer Autor als Mitläufer davon machen, weil letztlich im Entnazifizierungsverfahren nur wenige harte Fakten gegen ihn sprachen und sicher auch, weil die Nachkriegsgesellschaft mit ihrem Pragmatismus es so wollte.

Letztlich muss auch beim Blick auf die Literaturgeschichte genau hingesehen werden, müssen die Zwischentöne und Graubereiche ausgeleuchtet und jeder einzelne Fall differenziert betrachtet werden. Nur so wird ein Bild entstehen, das in der Lage ist, der Welt, die es erfassen soll, möglichst nahe zu kommen.

\section{Quellen und Literatur}

Adam, Christian (2016): Der Traum vom Jahre Null. Autoren, Bestseller, Leser: Die Neuordnung der Bücherwelt in Ost und West nach 1945. Berlin: Galiani.

Adam, Christian (2010): Lesen unter Hitler. Autoren, Bestseller, Leser im Dritten Reich. Berlin: Galiani.

Delius, Friedrich Christian (2018): Die Tücken des autobiographischen Erzählens. In: Sprache im technischen Zeitalter 225, S. 4-15.

Fritzlar, Sigrid (2011): Ehm Welk und der beschwerliche Aufbau der Volkshochschule Schwerin vor 65 Jahren. Archivalie des Monats Mai 2011. In: Landesarchiv Mecklenburg-Vorpommern (hrsg.): Archivalie des Monats. https://www.kulturwerte-mv.de/Landesarchiv/Archivalien/Bisherige-Beitr\%C3\%A4ge/2011-05-Ehm-Welk-und-die-Volkshochschule-Schwerin/ (22. 9. 2019).

25 Delius, Friedrich Christian (2018): Die Tücken des autobiographischen Erzählens. In: Sprache im technischen Zeitalter 225, S. 13 . 
Gruschka, Bernd R. (1995): Der gelenkte Buchmarkt. Die amerikanische Kommunikationspolitik in Bayern und der Aufstieg des Verlages Kurt Desch 1945 bis 1959. In: Archiv für die Geschichte des Buchwesens 44.

Löffler, Dietrich (2011): Buch und Lesen in der DDR. Ein literatursoziologischer Rückblick. Berlin: Ch. Links.

Reich, Konrad (2008): Ehm Welk. Der Heide von Kummerow. Die Zeit. Das Leben. Rostock: Hinstorff.

Reich-Ranicki, Marcel (2003): Mein Leben. München: dtv.

Reichsschrifttumskammer (hrsg.) (1935): Liste [1] des schädlichen und unerwünschten Schrifttums. Berlin: Reichsdruckerei.

Schenzinger, Karl Aloys (1949): Anilin. Roman. München: Wilhelm Andermann.

Schenzinger, Karl Aloys (1937): Anilin. Roman. Berlin: Zeitgeschichte-Verlag Wilhelm Andermann.

Schenzinger, Karl Aloys (1932): Der Hitlerjunge Quex. Berlin: Zeitgeschichte-Verlag Wilhelm Andermann.

The Visiting Committee of American Book Publishers (hrsg.) (1948): German Book Publishing and Allied Subjects. Munich - New York: [ohne Verlag].

Welk, Ehm (1943): Die Gerechten von Kummerow. Roman. Berlin: Deutscher Verlag.

Welk, Ehm (1937): Die Heiden von Kummerow. Roman. Berlin: Ullstein [später: Deutscher Verlag].

\section{Archivalische Quellen}

Bundesarchiv, Standort Berlin: BArch, DR 1/16217.

Staatsarchiv Landshut: Spruchkammerakten zu Karl Aloys Schenzinger, Spruchkammer Landau Nr. 2664.

Ullstein Archiv [Berlin]: Deutscher Verlag, Bericht über wichtige Geschäftsvorfälle im Oktober 1944.

Dr. Christian Adam / Christian1Adam@bundeswehr.org

Zentrum für Militärgeschichte und Sozialwissenschaften der Bundeswehr, Zeppelinstraße 127/128, 14471 Potsdam, D 